\title{
The Diagnostic Role of Brain MRI in Detection of Multiple Sclerosis Related Cognitive Impairment
}

\author{
Mohamed Saad', Maha Bilal'2, Wael Gabr ${ }^{1,3^{*}}{ }^{(\mathbb{C}}$, Aymen Abd Elnaby $^{1}$ \\ ${ }^{1}$ Neurology Department, Mansoura University, Mansoura, Egypt \\ ${ }^{2}$ Radiology Department, Mansoura University, Mansoura, Egypt \\ ${ }^{3}$ Internal Medicine Department, Tiabah University, Madina, KSA \\ Email: *waaael@yahoo.com, ${ }^{*}$ waaael@gmail.com
}

How to cite this paper: Saad, M., Bilal, M., Gabr, W. and Elnaby, A.A. (2019) The Diagnostic Role of Brain MRI in Detection of Multiple Sclerosis Related Cognitive Impairment. Journal of Behavioral and Brain Science, 9, 313-324.

https://doi.org/10.4236/jbbs.2019.98023

Received: July 7, 2019

Accepted: August 9, 2019

Published: August 12, 2019

Copyright $\odot 2019$ by author(s) and Scientific Research Publishing Inc. This work is licensed under the Creative Commons Attribution International License (CC BY 4.0).

http://creativecommons.org/licenses/by/4.0/

\begin{abstract}
Background: Cognitive impairment (CI) is a common manifestation of multiple sclerosis (MS), which can severely affect patients' and their families' life. Early suspicion and detection of CI can improve general medical management of MS patients. Objectives: To correlate MS related CI to cortical brain lesions using brain magnetic resonance imaging (MRI). Materials and Methods: Cognitive impairment was detected using mini mental state examination (MMSE); Neurological examination and brain MRI were performed for all patients. Correlation was calculated between disease cortical burden detected by MRI and CI. Results: Fifty-three patients with proven MS were scanned by brain MRI; $69.8 \%$ of them had cognitive impairment diagnosed with MMSE. The presence and severity of cognitive impairment was correlated to cortical brain lesion. Cognitive impairment was not correlated with non-cortical brain lesions or neurological physical disability measured by Expanded Disability Status Scale (EDSS). Conclusions: Presence of brain frontal cortical lesions detected by MRI in MS patients can predict subsequent development of MS-related CI.
\end{abstract}

\section{Keywords}

Multiple Sclerosis, Cognitive Impairment, MRI, Cortical Brain Lesion, MS Related CI

\section{Introduction}

In 1849, Dr. Friedrich von Frerichs was the primary one to note that multiple sclerosis (MS) isn't exclusively motor dysfunction but moreover ends up in cog- 
nitive impairment (CI) and he was the first one to document MS-related CI [1].

About $50 \%$ of MS patients will experience CI in the course of their disease, which commonly includes long-term memory defect rather than difficulty in making new memories, attention deficits, executive functioning impairment as well as delayed and inefficient information processing [2].

These MS-related CIs are less severe than CI observed in Alzheimer's disease; however, it can severely affect the patients and their family's lives [2]. It mainly leads to occupational disability as more than $50 \%$ of MS patients are unemployed within 10 years of diagnosis and this is mainly due to CI rather than to physical disability [3]. Also those patients became socially less active than before which also attributed mainly to MS-related CI [4]. So early detection and diagnosis of $\mathrm{CI}$ in MS patients is very important.

MRI is the cornerstone to the initial diagnostic workup of patients suspected to have MS [5] but MRI usually shows CNS lesions of variable sizes, numbers and in different locations among MS patients, moreover brain atrophy correlates moderately with MS-related CI so it can't be used solely to predict CI in MS patients [6] [7] [8] [9].

Correlating the exact site of the cerebral MS lesions to CI may give us a sight for early detection of $\mathrm{CI}$, which has important implications for managing and compensating for the daily problems that $\mathrm{CI}$ may produce, as well as to reveal areas of the brain that might be logical therapeutic targets for management and research. The Objectives of our study is to correlate between the exact site of demyelinating lesions detected by brain MRI and CI in MS patients.

\section{Materials and Methods}

After approval from our institutional ethical committee, this study was conducted throughout the amount from August 2016 until December 2018, in line with the rules of 1995 of Helsinki.

A fifty three consecutive patients with confirmed diagnosis of MS according to Polman and his colleagues 2011 [10] who revised neurology outpatient clinics at Mansoura University Hospital, were enrolled in this study, 33 of them were females and 20 were males. A full detailed history taking and complete neurological examination was performed by a specialized neurologist for each patient.

Inclusion criterion was a diagnosis of MS and the patient agree to gave their informed consent to participate in this study this study. Exclusion criteria were patients unfit to perform brain MRI for any reasons as prosthetic heart pacemaker or patients who have a metallic foreign body (metal sliver) in their eye, or who have an aneurysm clip in their brain.

According to disease progression, patients were sub-grouped into; relapsing remitting (RR) [24 patients], primary progressive (PP) [13 patients], secondary progressive (SP) [9 patients] and 7 patients were belonging to clinically isolated syndrome (CIS) group.

Mini mental state examination (MMSE) was used to detect cognitive impair- 
ment (CI) among studied MS patients, Scores of 25 - 30 out of 30 are considered normal; the National Institute for Health and Care Excellence (NICE) classifies 21 - 24 as mild CI, 10 - 20 as moderate CI and $<10$ as severe cognitive impairment [11]. Any patient received elicited drugs, steroids or any psychoactive therapy during the last 3 months, that can influence cognitive test outcome of the patient was excluded from this study, also patients with an acute relapsing phase of MS were excluded.

The patients' physical disabilities were assessed by the Expanded Disability Status Scale (EDSS) which ranges from 0 to 10 in 0.5-unit increments (Appendix). It is based on measures of impairment in 8 functional systems (pyramidal, cerebellar, brainstem, sensory, sphincter, visual, mental and other functions). Each functional system is scored on a scale of 0 (no disability) to 5 or 6 (increasing disability). EDSS from 1.0 to 4.5 indicate MS patients who are able to walk without aid; while score 5.0 to 9.5 indicates MS patients with impaired walking [12].

All patients were radiologically evaluated by brain MRI, The MRI examinations were performed on 1.5 Tesla superconducting magnet (Philips Interna) with the patient in the supine position using a standard head coil of $8-16$ channels. Routine T1 weighted image (T1WI), T2 weighted image (T2WI) and Fluid-attenuated inversion recovery (FLAIR) images were done in all patients. First a scout sagittal T1-WI was obtained followed by axial T1-WI (TR/TE $=500 / 14$ $\mathrm{ms})$, then axial T2-WI (TR/TE $=4490 / 80 \mathrm{~ms})$ were obtained with FOV of $20-24$ $\mathrm{cm}$, matrix size of $256 \times 256$, slice thickness of $5 \mathrm{~mm}$ and interslice gap of $1 \mathrm{~mm}$, followed by FLAIR images $(\mathrm{TR} / \mathrm{TE}=10.000 / 120 \mathrm{~ms}), \mathrm{TI}=2.500 \mathrm{msec}, \mathrm{ETL}=$ 23.50. This was followed by DIR MRI sequences with $\mathrm{TR}=15.631 \mathrm{msec}, \mathrm{TE}=25$ msec, $\mathrm{TI}=3.400 \mathrm{msec}$, delay $=325 \mathrm{msec}, \mathrm{ETL}=17.50$ continuous axial slices, thickness $=3 \mathrm{~mm}$, matrix size $=130-256, \mathrm{FOV}=200-250 \mathrm{~mm}$. Three-dimensional Double inversion recovery (DIR) images were obtained with the following technical features; TR, +1000 ms, TE, 120 ms, TI, 2000 ms, matrix, $256 \times 149$, NEX, 2, slice thickness, $5 \mathrm{~mm}$, section width, $1 \mathrm{~mm}$ and exposure time, $1.30 \mathrm{~min}$.

The MRI was analyzed by expert neuroradiologist blinded for the clinical and paraclinical test results. All of the hyperintense signals detected in the T2WI, FLAIR and DIR images are considered as lesions.

The locations of the lesions were identified and their anatomical regions were divided into cortical and non-cortical brain lesions (juxtra-cortical, deep white matter, deep gray matter, peri ventricular white matter and infratentorial region), On DIR, particular attention was given to artifacts and cortical Lesions (CL) were defined as those lesions confined to cortical ribbon and not involving underling subcortical white matter. The lesions numbers according to regions were determined.

\section{Statistical analysis}

The statistical analysis was performed using IBM SPSS Statistics for Windows, Version 20.0. Armonk, NY: IBM Corp, 2011. The continuum was expressed in number and percentage. The difference among group was determined using 
one-way analysis of variance (t-test), for continuous data Chi square test for categorical data. The comparison between the patients with cortical and with non-cortical brain MRI abnormality was determined by independent sample t-test. Statistical significance was set at $\mathrm{P}<0.05$.

\section{Results}

Fifty-three patients with proven diagnosis of MS underwent brain MRI to localize the brain demyelinating lesions, $62.3 \%$ of them were females (33 patients) and $37.7 \%$ were males (20 patients), their ages ranged from 18 to 50 years with mean age of $33.81 \pm 7.87$ years, the mean duration of the disease was $37.75 \pm$ 23.24 months, twenty-four patients were belonging to the relapsing remitting type of MS, 13 patients were primary progressive, 9 were secondary progressive and only 7 were belonging to the clinically isolated syndrome category of MS (Table 1).

Brain MRI analysis of the cortical lesions revealed that, anatomically brain cortical demyelinating patches mainly located at frontal lobe (106 cortical lesions), followed by temporal lobe (56 cortical lesions), then parietal lobe (23 cortical lesions), then occipital lobe (13 cortical lesions) and lastly cerebellar region (8 cortical lesions); while clinically, brain cortical demyelinating patches mainly affect patients with relapsing remitting MS (108 cortical lesions), followed by patients with primary progressive MS (51 cortical lesions), then patients with secondary progressive MS (32 cortical lesions) and finally patients with clinically isolated syndrome (15 cortical lesions) (Table 2 ).

Table 1. Demographic data of the patients.

\begin{tabular}{|c|c|c|c|c|c|c|}
\hline & & Male & Female & Total & Mean \pm SD & Range \\
\hline \multicolumn{2}{|c|}{ Patient No. } & $20(37.7 \%)$ & $33(62.3 \%)$ & $53(100 \%)$ & & \\
\hline \multicolumn{2}{|c|}{ Age(years) } & $35.50 \pm 7.50$ & $32.79 \pm 8.02$ & & $33.81 \pm 7.87$ & $(18-50)$ \\
\hline \multicolumn{2}{|c|}{ Age of onset (years) } & $32.17 \pm 7.07$ & $29.76 \pm 7.26$ & & $30.65 \pm 7.22$ & $(21-48)$ \\
\hline \multicolumn{2}{|c|}{ Disease duration (months) } & $40.00 \pm 27.10$ & $36.39 \pm 20.89$ & & $37.75 \pm 23.24$ & $(3-120)$ \\
\hline \multicolumn{2}{|c|}{ EDSS } & $2.50 \pm 1.85$ & $1.03 \pm 1.63$ & & $1.58 \pm 1.84$ & $(0-6)$ \\
\hline \multirow{4}{*}{ MS Types } & $\mathrm{RR}$ & $10(18.9 \%)$ & $14(26.4 \%)$ & $24(45.3 \%)$ & & \\
\hline & PP & $5(9.4 \%)$ & $8(15.1 \%)$ & $13(24.5 \%)$ & & \\
\hline & SP & $3(5.7 \%)$ & $6(11.3 \%)$ & $9(17.0 \%)$ & & \\
\hline & CIS & $2(3.8 \%)$ & $5(9.4 \%)$ & $7(13.2 \%)$ & & \\
\hline \multirow{4}{*}{$\begin{array}{c}\text { Cognitive } \\
\text { Impairment }\end{array}$} & No & $4(7.5 \%)$ & $12(22.6 \%)$ & $16(30.2 \%)$ & & \\
\hline & Mild & $5(9.4 \%)$ & $16(30.2 \%)$ & $21(39.6 \%)$ & & \\
\hline & Moderate & $8(15.1 \%)$ & $3(5.7 \%)$ & $11(20.8 \%)$ & & \\
\hline & Severe & $3(5.7 \%)$ & $2(3.8 \%)$ & $5(9.4 \%)$ & & \\
\hline
\end{tabular}

No. $=$ number, $\mathrm{SD}=$ standard deviation, $\mathrm{MS}=$ multiple sclerosis, $\mathrm{RR}=$ relapsing remitting, $\mathrm{PR}=$ primary progressive, $\mathrm{SP}=$ secondary progressive, $\mathrm{CIS}=$ clinically isolated syndrome, EDSS = expanded disability status scale. 
When comparing cortical and non-cortical brain demyelinating lesions and correlating them to cognitive impairment it revealed that total burden of demyelinating lesions correlates more with the presence of cognitive impairment rather than the cortical lesions, except in frontal lobe where cortical brain lesions correlates significantly with cognitive impairment when compared with non-cortical brain lesions (Table 3)

Table 4 showing a correlation between the number of the cortical brain lesions and degree of cognitive impairment, it shows a high statistically significant relationship between the number of cortical brains demyelinating lesions and the degree of $\mathrm{CI}$ in different brain region except in the cerebellar region were such correlation didn't exist.

Table 2. The number of lesions detected by MRI DIR in different anatomical region among studied subgroups.

\begin{tabular}{cccccc}
\hline & RR & PP & SP & CIS & Total \\
\hline Frontal & $64(59.2 \%)$ & $23(43.1 \%)$ & $12(37.5 \%)$ & $7(46.6 \%)$ & 106 \\
Temporal & $25(23.1 \%)$ & $18(35.2 \%)$ & $9(28.1 \%)$ & $4(26.6 \%)$ & 56 \\
Parietal & $9(8.3 \%)$ & $5(9.8 \%)$ & $6(18.7 \%)$ & $3(20 \%)$ & 23 \\
Occipital & $5(4.6 \%)$ & $3(5.8 \%)$ & $4(12.5 \%)$ & $1(6.6 \%)$ & 13 \\
Cerebellar & $5(4.6 \%)$ & $2(3.9 \%)$ & $1(3.1 \%)$ & $0(0.0 \%)$ & 8 \\
Total & $108(100 \%)$ & $51(100 \%)$ & $32(100 \%)$ & $15(100 \%)$ & 206
\end{tabular}

No. $=$ number, $\mathrm{SD}=$ standard deviation, $\mathrm{MRI}=$ magnetic resonant imaging, $\mathrm{DIR}=$ double inversion recovery, $\mathrm{MS}=$ multiple sclerosis, $\mathrm{RR}=$ relapsing remitting, $\mathrm{PR}=$ primary progressive, $\mathrm{SP}=$ secondary progressive, $\mathrm{CIS}=$ clinically isolated syndrome.

Table 3. Statistical comparisons between cortical and non-cortical lesions in patients with Cognitive impairment according to lesion locations.

\begin{tabular}{lccccc}
\hline & Frontal Lobe & Temporal Lobe & Parietal Lobe & Occipital Lobe & Cerebellar Lobe \\
\hline $\begin{array}{l}\text { CL versus } \\
\text { Non-CL }\end{array}$ & $\mathrm{P}=0.001^{*}$ & $\mathrm{P}=0.201$ & $\mathrm{P}=0.706$ & $\mathrm{P}=0.393$ & $\mathrm{P}=0.065$ \\
\hline
\end{tabular}

$\mathrm{CL}=$ presence of cortical brain lesion, Non- $\mathrm{CL}=$ non cortical brain lesions, ${ }^{*}=$ statistically significant difference.

Table 4. The relation between the number of cortical cortical lesions and the degree of cognitive impairment in different studied areas.

\begin{tabular}{ccccc}
\hline & Mild CI & Moderate CI & Severe CI & \multirow{2}{*}{ P-Value } \\
\cline { 2 - 4 } & Mean \pm SD & Mean \pm SD & Mean \pm SD & \\
\hline Frontal Lobe & $1.57 \pm 1.16$ & $2.55 \pm 1.21$ & $6.00 \pm 1.58$ & $\mathrm{P}<0.05^{*}$ \\
Temporal Lobe & $0.52 \pm 0.81$ & $1.55 \pm 0.52$ & $3.40 \pm 1.14$ & $\mathrm{P}<0.05^{*}$ \\
Parietal Lobe & $0.19 \pm 0.6$ & $0.45 \pm 0.52$ & $1.80 \pm 0.84$ & $\mathrm{P}<0.05^{*}$ \\
Occipital Lobe & $0.14 \pm 0.48$ & $0.09 \pm 0.3$ & $1.40 \pm 0.55$ & $\mathrm{P}<0.05^{*}$ \\
Cerebellar Lobe & $0.05 \pm 0.22$ & $0.18 \pm 0.4$ & $1.00 \pm 0.71$ & $\mathrm{P}=0.094^{* *}$ \\
\hline
\end{tabular}

$\mathrm{CI}=$ cognitive impairment, $\mathrm{SD}=$ standard deviation, ${ }^{*}=$ statistically significant, ${ }^{*}=$ statistically insignificant. 


\section{Discussion}

Early recognition of MS patients susceptible to develop cognitive impairment for early detection and management of cognitive impairment may improve the total medical care applied to MS patients, so we try to investigate the relation between cognitive impairment and abnormal brain demyelinating lesions detected by brain MRI; and comparing between cortical brain lesions and non-cortical brain lesions in different brain lesion to cognitive impairment.

In this study we find a relationship between cortical brain demyelination lesions and cognitive impairment especially in frontal lobe when compared with non-cortical lesions. This means that during clinical practice radiological detection of brain cortical demyelination lesions in MS patients raise the susceptibility for subsequent development of cognitive impairment, many other studies also confirm our results and highlighted the effect of cortical brain lesions on cognition among MS patients [13] [14].

Analysis of our date revealed that; not only presence of cortical brain lesions correlated with the occurrence of cognitive impairment but also the number of cortical brain lesions directly correlate with the severity of cognitive impairment, these results was in a harmony with the results obtained by Calabrese and his colleague who concluded that the degree of cognitive impairment in patients with MS related to the extent of brain cortical lesions [15] and in agreement with Rinaldi and his colleague who concluded that cortical lesions burden correlates with the severity of MS-related CI [16], also same results was obtained by Nelson and his colleague as they concluded that the size of brain cortical lesions affect the degree of CI [17].

In this study, comparing the cortical lesions in different brain regions and correlating them to cognitive impairment revealed that frontal cortical demyelinating lesions show statistically significant correlation with the MS-related cognitive impairment, this finding confirmed by the resulted obtained by Sun and his colleague as they confirm that brain disease in the frontal lobe related to the degree of cognitive impairment in MS patients [18].

In our studied MS subgroups, the cortical lesions were more frequent in relapsing remitting MS patients, this may be explained by frequent attacks and longer duration of the disease, in a study conducted by Calabrese et al., the number of cortical lesion associated with $\mathrm{CI}$ is more in relapsing remitting MS patients [19], in contrary to our results; other studies found that progressive MS diseases more vulnerable to cognitive impairment compared to relapsing remitting MS [20] [21] [22].

\section{Conclusion}

This study revealed that cortical brain lesions detected by MRI can be used as marker for subsequent development of cognitive impairment, especially if these cortical brain lesions are detected in the frontal lobe. 


\section{Acknowledgements}

We wish to express our great appreciation to our patients and their family for supporting us during this work.

\section{Ethics Approval and Consent to Participate}

The study protocol was approved by the ethical committee in Mansoura University Hospital, AL Mansoura, Egypt, on September 2016. Participation was voluntary and all contributors or their first-degree relatives received detailed information about the aims of this research work and an informed consent was obtained prior to the commencement of the study.

\section{Consent for Publication}

A written informed consent for the publication was obtained from all the participants and their first-degree relatives.

\section{Availability of Data and Materials}

All raw data will be available on the editor request.

\section{Conflicts of Interest}

The authors declare that they have no competing interest

\section{Funding}

This research did not receive any grant from funding agencies in the public, commercial, or not-for-profit sectors.

\section{Authors' Contributions}

This work design done mainly by authors MS. All other authors reviewed literature, and wrote the primary draft of the manuscript, $\mathrm{MB}$ collected the radiological data, WG write the primary manuscript \& performed statistical analysis. All authors read and approved the final manuscript.

\section{References}

[1] Jambor, K.L. (1969) Cognitive Functioning in Multiple Sclerosis. The British Journal of Psychiatry, 115, 765-775. https://doi.org/10.1192/bjp.115.524.765

[2] Chiaravalloti, N.D. and Deluca, J. (2008) Cognitive Impairment in Multiple Sclerosis. The Lancet Neurology, 7, 1139-1151. https://doi.org/10.1016/S1474-4422(08)70259-X

[3] Julian, L.J., Vella, L., Vollmer, T., Hadjimichael, O. and Mohr, D.C. (2008) Employment in Multiple Sclerosis. Exiting and Re-Entering the Work Force. Journal of Neurology, 255, 1354-1360. https://doi.org/10.1007/s00415-008-0910-y

[4] Larocca, N., Kalb, R., Scheinberg, L. and Kendall, P. (1985) Factors Associated with Unemployment of Patients with Multiple Sclerosis. Journal of Chronic Diseases, 38, 203-210. https://doi.org/10.1016/0021-9681(85)90093-1 
[5] Kacar, K., Rocca, M.A., Copetti, M., Sala, S., Mesaros, S., Stosic, O.T., et al. (2011) Overcoming the Clinical-MR Imaging Paradox of Multiple Sclerosis: MR Imaging Data Assessed with a Random Forest Approach. American Journal of Neuroradiology, 32, 2098-2102. https://doi.org/10.3174/ajnr.A2864

[6] Zivadinov, R., Sepcic, J., Nasuelli, D., De, M.R., Bragadin, L.M., Tommasi, M.A., et al. (2001) A Longitudinal Study of Brain Atrophy and Cognitive Disturbances in the Early Phase of Relapsing-Remitting Multiple Sclerosis. Journal of Neurology, Neurosurgery, and Psychiatry, 70, 773-780. https://doi.org/10.1136/jnnp.70.6.773

[7] Christodoulou, C., Krupp, L.B., Liang, Z., Huang, W., Melville, P., Roque, C., et al. (2003) Cognitive Performance and MR Markers of Cerebral Injury in Cognitively Impaired MS Patients. Neurology, 60, 1793-1798.

https://doi.org/10.1212/01.WNL.0000072264.75989.B8

[8] Sanfilipo, M.P., Benedict, R.H., Weinstock-Guttman, B. and Bakshi, R. (2006) Gray and White Matter Brain Atrophy and Neuropsychological Impairment in Multiple Sclerosis. Neurology, 66, 685-692.

https://doi.org/10.1212/01.wnl.0000201238.93586.d9

[9] Sanchez, M.P., Nieto, A., Barroso, J., Martin, V. and Hernandez, M.A. (2008) Brain Atrophy as a Marker of Cognitive Impairment in Mildly Disabling Relapsing-Remitting Multiple Sclerosis. European Journal of Neurology, 15, 1091-1099. https://doi.org/10.1111/j.1468-1331.2008.02259.x

[10] Polman, C.H., Reingold, S.C., Banwell, B., Clanet, M., Cohen, J.A., Filippi, M., et al. (2011) Diagnostic Criteria for Multiple Sclerosis: 2010 Revisions to the McDonald Criteria. Annals of Neurology, 69, 292-302. https://doi.org/10.1002/ana.22366

[11] Folstein, M.F., Folstein, S.E. and McHugh, P.R. (1975) Mini-Mental State. A Practical Method for Grading the Cognitive State of Patients for the Clinician. Journal of Psychiatric Research, 12, 189-198. https://doi.org/10.1016/0022-3956(75)90026-6

[12] Kurtzke, J.F. (1955) A New Scale for Evaluating Disability in Multiple Sclerosis. Neurology, 5, 580-583. https://doi.org/10.1212/WNL.5.8.580

[13] Vercellino, M., Plano, F., Votta, B., Mutani, R., Giordana, M.T. and Cavalla, P. (2005) Grey Matter Pathology in Multiple Sclerosis. Journal of Neuropathology \& Experimental Neurology, 64, 1101-1117. https://doi.org/10.1097/01.jnen.0000190067.20935.42

[14] Kutzelnigg, A. and Lassmann, H. (2006) Cortical Demyelination in Multiple Sclerosis: A Substrate for Cognitive Deficits? Journal of the Neurological Sciences, 245, 123-126. https://doi.org/10.1016/j.jns.2005.09.021

[15] Calabrese, M., Rinaldi, F., Grossi, P. and Gallo, P. (2011) Cortical Pathology and Cognitive Impairment in Multiple Sclerosis. Expert Review of Neurotherapeutics, 11, 425-432. https://doi.org/10.1586/ern.10.155

[16] Rinaldi, F., Calabrese, M., Grossi, P., Puthenparampil, M., Perini, P. and Gallo, P. (2010) Cortical Lesions and Cognitive Impairment in Multiple Sclerosis. Neurological Sciences, 31, S235-S237. https://doi.org/10.1007/s10072-010-0368-4

[17] Nelson, F., Datta, S., Garcia, N., Rozario, N.L., Perez, F., Cutter, G., et al. (2011) Intracortical Lesions by 3T Magnetic Resonance Imaging and Correlation with Cognitive Impairment in Multiple Sclerosis. Multiple Sclerosis Journal, 17, 1122-1129. https://doi.org/10.1177/1352458511405561

[18] Sun, Y., Cao, W., Ding, W., Wang, Y., Han, X., Zhou, Y., et al. (2016) Cerebral Blood Flow Alterations as Assessed by 3D ASL in Cognitive Impairment in Patients with Subcortical Vascular Cognitive Impairment: A Marker for Disease Severity. Frontiers in Aging Neuroscience, 8, 211. https://doi.org/10.3389/fnagi.2016.00211 
[19] Calabrese, M., Agosta, F., Rinaldi, F., Mattisi, I., Grossi, P., Favaretto, A., et al. (2009) Cortical Lesions and Atrophy Associated with Cognitive Impairment in Relapsing-Remitting Multiple Sclerosis. Archives of Neurology, 66, 1144-1150. https://doi.org/10.1001/archneurol.2009.174

[20] Roosendaal, S.D., Moraal, B., Pouwels, P.J., Vrenken, H., Castelijns, J.A., Barkhof, F., et al. (2009) Accumulation of Cortical Lesions in MS: Relation with Cognitive Impairment. Multiple Sclerosis Journal, 15, 708-714. https://doi.org/10.1177/1352458509102907

[21] Thornton, A.E. and Raz, N. (1997) Memory Impairment in Multiple Sclerosis: A Quantitative Review. Neuropsychology, 11, 357-366. https://doi.org/10.1037/0894-4105.11.3.357

[22] Camp, S.J., Stevenson, V.L., Thompson, A.J., Miller, D.H., Borras, C., Auriacombe, S., et al. (1999) Cognitive Function in Primary Progressive and Transitional Progressive Multiple Sclerosis: A Controlled Study with MRI Correlates. Brain, 122, 1341-1348. https://doi.org/10.1093/brain/122.7.1341 


\section{Appendix}

Expanded Disability Status Scale (EDSS) [12].

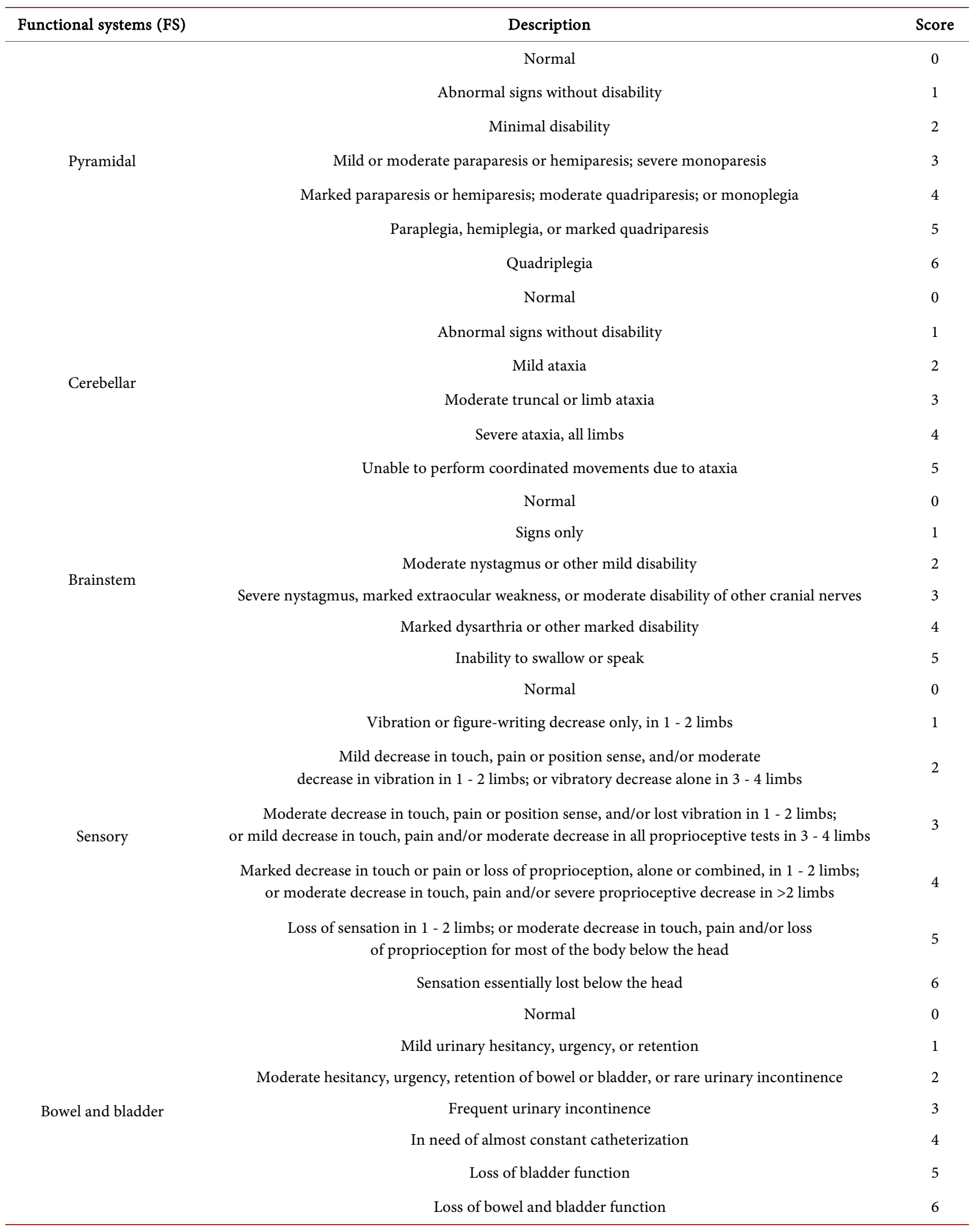


Visual

Cerebral

Other

$$
\text { Normal }
$$

Scotoma with visual acuity (corrected) better than 20/30

Worse eye with scotoma with maximal visual acuity (corrected) of 20/30 to 20/59

Worse eye with large scotoma, or moderate decrease in fields, but with maximal visual acuity (corrected) of 20/60 to 20/99

Worse eye with marked decrease of fields and maximal visual acuity (corrected) of 20/100 - 20/200; grade 3 plus maximal acuity of better eye of $\leq 20 / 60$

Worse eye with maximal visual acuity (corrected) less than 20/200; grade 4 plus maximal acuity of better eye of $20 / 60$ or less

Grade 5 plus maximal visual acuity of better eye of $20 / 60$ or less 6

Normal 0

Mood alteration only (Does not affect DSS score) 1

Mild decrease in mentation $\quad 2$

Moderate decrease in mentation 3

Marked decrease in mentation (chronic brain syndrome-moderate) 4

Dementia or chronic brain syndrome-severe or incompetent $\quad 5$

Any other neurologic findings attribugted to MS

$0=$ Normal neurologic exam (all grade 0 in Functional Systems [FS]; Cerebral grade 1 acceptable).

1.0 = No disability, minimal signs in one FS (i.e. grade 1 excluding Cerebral grade 1).

$1.5=$ No disability minimal signs in more than one FS (more than one grade 1 excluding Cerebral grade 1).

$2.0=$ Minimal disability in one FS (one FS grade 2, others 0 or 1 ).

$2.5=$ Minimal disability in two FS (two FS grade 2 , others 0 or 1 ).

3.0 = Moderate disability in one FS (one FS grade 3, others 0 or 1), or mild disability in three or four FS (three/four FS grade 2, others 0 or 1) though fully ambulatory.

3.5 = Fully ambulatory but with moderate disability in one FS (one grade 3 ) and one or two FS grade 2; or two FS grade 3; or five FS grade 2 (others 0 or 1).

4.0 = Fully ambulatory without aid, self-sufficient, up and about some 12 hours a day despite relatively severe disability consisting of one FS grade 4 (others 0 or 1), or combinations of lesser grades exceeding limits of previous steps. Able to walk without aid or rest some 500 meters.

4.5 = Fully ambulatory without aid, up and about much of the day, able to work a full day, may otherwise have some limitation of full activity or require minimal assistance; characterized by relatively severe disability, usually consisting of one FS grade 4 (others 0 or 1 ) or combinations of lesser grades exceeding limits of previous steps. Able to walk without aid or rest for some 300 meters. 
5.0 = Ambulatory without aid or rest for about 200 meters; disability severe enough to impair full daily activities (e.g. to work full day without special provisions). (Usual FS equivalents are one grade 5 alone, others 0 or 1; or combinations of lesser grades usually exceeding specifications for step 4.0).

5.5 = Ambulatory without aid or rest for about 100 meters; disability severe enough to preclude full daily activities (Usual FS equivalents are one grade 5 alone, others 0 or 1 ; or combinations of lesser grades usually exceeding those for step 4.0).

$6.0=$ Intermittent or unilateral constant assistance (cane, crutch, or brace) required to walk about 100 meters with or without resting (Usual FS equivalents are combinations with more than two FS grade $3+$ ).

6.5 = Constant bilateral assistance (canes, crutches, or braces) required to walk about 20 meters without resting (Usual FS equivalents are combinations with more than two FS grade 3+).

$7.0=$ Unable to walk beyond about 5 meters even with aid, essentially restricted to wheelchair; wheels self in standard wheelchair and transfers alone; up and about in w/c some 12 hours a day (Usual FS equivalents are combinations with more than one FS grade 4; very rarely, pyramidal grade 5 alone).

$7.5=$ Unable to take more than a few steps; restricted to wheelchair; may need aid in transfer; wheels self but cannot carry on in standard wheelchair a full day; may require motorized wheelchair (Usual FS equivalents are combinations with more than one FS grade $4+$ ).

$8.0=$ Essentially restricted to bed or chair or perambulated in wheelchair, but may be out of bed itself much of the day; retains many self-care functions; generally has effective use of arms (Usual FS equivalents are combinations, generally grade $4+$ in several systems).

$8.5=$ Essentially restricted to bed much of the day; has some effective use of $\operatorname{arm}(\mathrm{s})$; retains some self-care functions (Usual FS equivalents are combinations, generally $4+$ in several systems).

9.0 = Helpless bed patient; can communicate and eat (Usual FS equivalents are combinations, mostly grade $4+$ ).

9.5 = Totally helpless bed patient; unable to communicate effectively or eat/swallow (Usual FS equivalents are combinations, almost all grade $4+$ ).

$10.0=$ Death due to MS 